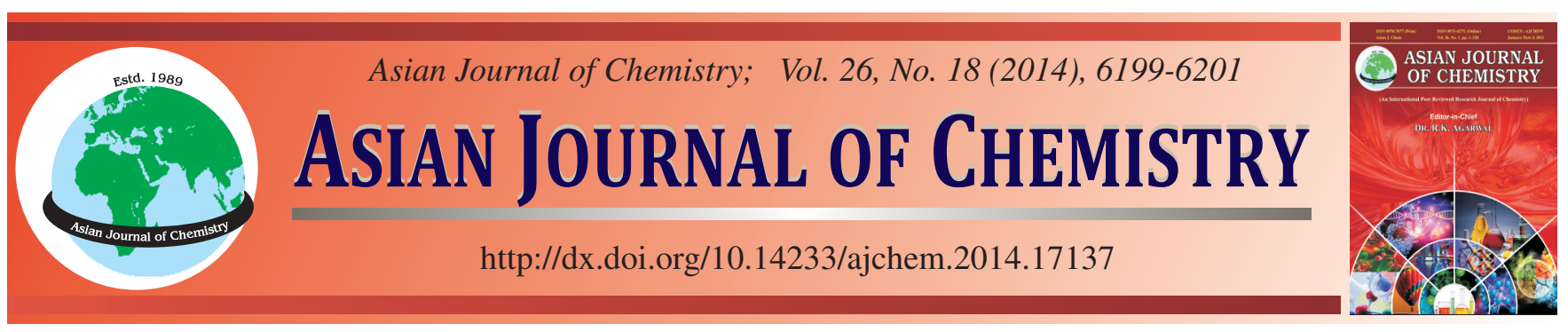

\title{
Synthesis and Structure Optimization of Schiff Base Derived from $p$-Chlorobenzaldehyde and $p$-Aminobenzoic Acid by Rapid Efficient Gas Impacting Reaction
}

\author{
YAN-HuA CAI
}

Chongqing Key Laboratory of Environmental Materials \& Remediation Technologies, Chongqing University of Arts and Sciences, Yongchuan, Chongqing 402160, P.R. China

Corresponding author: E-mail: caiyh651@aliyun.com

To confirm the advantage for synthesis of Schiff base using jet milling, Schiff base derived from $p$-chlorobenzaldehyde and $p$-aminobenzoic acid was synthesized using jet milling. The ${ }^{1} \mathrm{H}$ NMR and FTIR technology confirmed the structure of Schiff base. Optimized geometrical structure showed that the HOMO and LUMO of Schiff base were $-9.16 \mathrm{eV}$ and $-1.256 \mathrm{eV}$, respectively. And that the HOMO of Schiff base focused on benzene, in contrast, the LUMO of Schiff base was dispersed. The bond length and bond angle between atoms at imine group was smaller than that of other group.

Keywords: Schiff base, Jet milling, Geometrical structure, $p$-Aminobenzoic acid.

ᄂ - - - - - - - - - - - - - - - - - - - - - - - - - - - - - - - -

\section{INTRODUCTION}

Continuous development of science and technology have made the production materials become more and more fine due to better performance of fine materials. Jet milling, as an important grinding method, has been developed to widely used in materials processing field ${ }^{1}$. Fine and superfine materials without any contamination are obtained using jet milling, furthermore, the cost of jet milling is very acceptable. Mcguiness et $a$. $^{2}$ reported that hydrogen decrepotation and jet milling was used to produce powder for the processing of permanent magnets. These results showed that the procedure is very successful and the Nd-Fe-B and Nd-Dy-Fe-Nb-B alloys prepared by hydrogen decrepotation and jet milling showed excellent coercivities. Similarly, micro-fine high Nb-containing TiAl alloyed powders were made using jet milling. However, the paper focus on the effect of classifier frequency on powder characteristics, the size of particle decreased with increasing of classifier frequency and the size of powder is lower than 25 $\mu \mathrm{m}$ at a classifier frequency higher than $38 \mathrm{~Hz}^{3}$.

The comminution rate of diatomaceous earth comminuted using jet milling increased with increasing in the pressure of pulverizing-air. However, the average size and comminution rate of diatomaceous earth decreased with increasing comminution time ${ }^{4}$. However, the ultra-fine materials using jet milling is not necessarily the best. Saleem and Smyth ${ }^{5}$ reported that micronization of a soft material using jet milling might not be possible to achieve particle size of approximately $5 \mu \mathrm{m}$ for pulmonary delivery.
On the other hand, jet milling with great energy during materials process can cause chemical reaction between different reactants occure. Thus, jet milling has been tried to apply in chemical reaction field ${ }^{6,7}$, Cai et al ${ }^{8}$ reported synthesis of three Schiff bases derived from $p$-aminobenzoic acid. The results showed that the reaction using jet milling not only involved short reaction time and simple operation, but also gave high yield.

In this paper, to further confirm advantage of synthesis Schiff base using jet milling, the reaction using jet milling still focus on the synthesis of Schiff base compound with important application value. Schiff base derived from $p$-chloroben-zaldehyde and $p$ aminobenzoic acid was synthesized by jet milling, the structure of Schiff base was characterized by ${ }^{1} \mathrm{H}$ NMR and FTIR and structure optimization also was investigated.

\section{EXPERIMENTAL}

Materials and reaction equipment: The $p$-aminobenzoic acid and $p$-chlorobenzaldehyde were purchased from Mianyan Rongshen Chemical Reagents Company (Sichuan Province, China) and Beijing Chemical Reagents Company (Beijing, China), respectively. The modified jet milling is showed in Fig. 1.

Synthesis of Schiff base: Schiff base derived from $p$ chlorobenzaldehyde and $p$-aminobenzoic acid was prepared as following in Fig. 2 and the preparation process is similar to that of our previous paper ${ }^{6}$. 


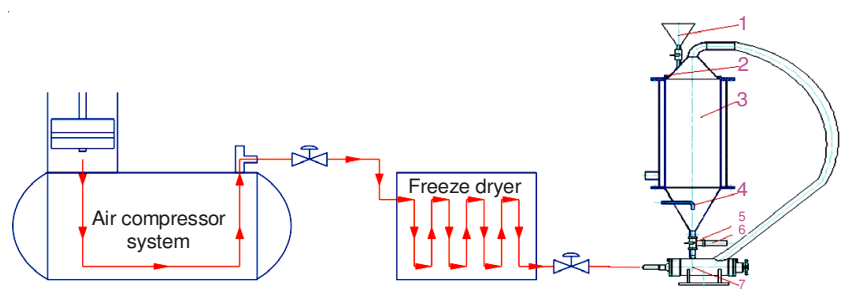

Fig. 1. Schematic drawing of the reaction

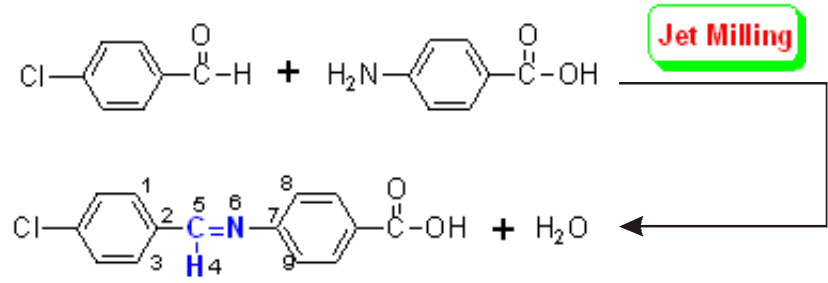

Fig. 2. Synthesis of Schiff base

The structure of Schiff base was characterized by ${ }^{1} \mathrm{H}$ NMR and FTIR. The ${ }^{1} \mathrm{H}$ nuclear magnetic resonance was recorded on Brucker AVANCE 300 spectrometers and the infrared spectra were measured on Nicolet 380 FT-IR spectrometer, in the range $4000-400 \mathrm{~cm}^{-1}$.

\section{RESULTS AND DISCUSSION}

Structure of Schiff base: The ${ }^{1} \mathrm{H}$ NMR of Schiff base derived from $p$-chlorobenzaldehyde and $p$-aminobenzoic acid was shown in Fig. 3. The multiple peaks at $\delta_{\mathrm{H}}=7.33-8$ belonged to benzene proton resonance peaks, the single peak at $\delta_{\mathrm{H}}=$ 8.66 was $\mathrm{CH}=\mathrm{N}$ proton resonance peak and the peak at $\delta_{\mathrm{H}}=$ 12.32 contributed to $\mathrm{COOH}$ proton resonance. NMR analysis confirmed that Schiff base derived from $p$-chlorobenzaldehyde and $p$-aminobenzoic acid was synthesized.

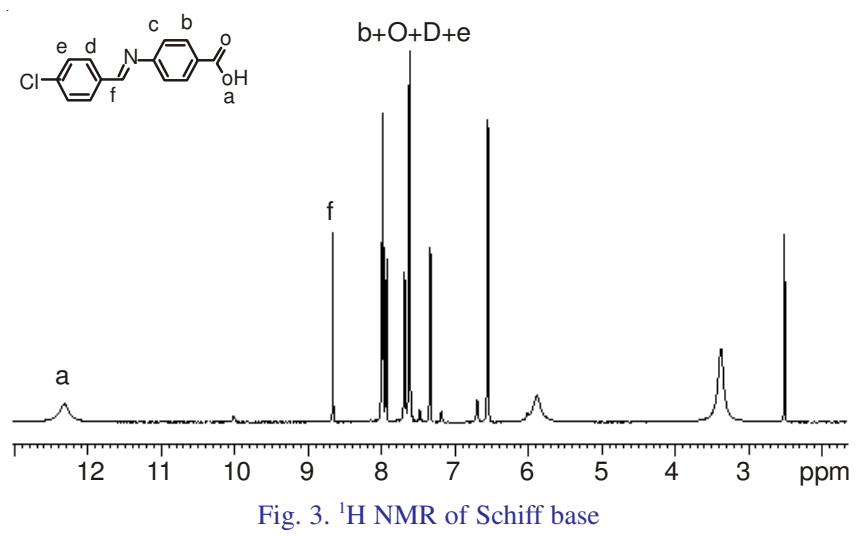

In addition, the structure of Schiff base was further confirmed by FTIR. Fig. 4 showed the FTIR spectra of Schiff base derived from $p$-chlorobenzaldehyde and $p$-aminobenzoic acid, it is clear form Fig. 4 that the peaks at $3364.1 \mathrm{~cm}^{-1}$ belonged to $\mathrm{O}-\mathrm{H}$ stretching vibration absorption, the absorption peak at $1681.4 \mathrm{~cm}^{-1}$ contributed to $\mathrm{C}=\mathrm{O}$ stretching vibration of $\mathrm{COOH}$, the absorption peak at $1627.7 \mathrm{~cm}^{-1}$ contributed to $\mathrm{C}=\mathrm{N}$ stretching vibration due to conjugated effect. The absorption peaks at $1592.9,1566.5,1488.8$ and $1423.6 \mathrm{~cm}^{-1}$ proved benzene. The absorption peak at $1167.9 \mathrm{~cm}^{-1}$ contribute to $\mathrm{C}-\mathrm{O}$

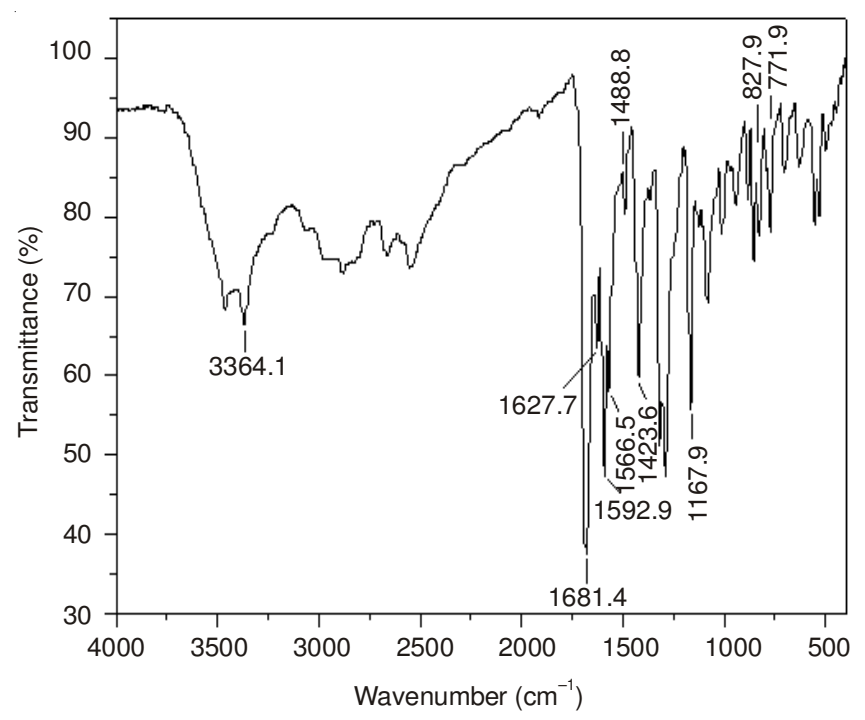

Fig. 4. FTIR of Schiff base

stretching vibration, the absorption peak at 827.9 and 771.9 $\mathrm{cm}^{-1}$ belongs to $\mathrm{C}-\mathrm{H}$ deformation vibration of benzene.

Structure optimization of Schiff base: Structure optimization of Schiff base derived from $p$-chlorobenzaldehyde and $p$-aminobenzoic acid was carried out using the program VAMP of Materials Studio software. The optimized geometric structure of Schiff base was shown in Fig. 5. Meantime, the frontier molecular orbital energy was obtained by theoretical calculation via VAMP. The value of frontier molecular orbital energy was following: the highest occupied molecular orbital (HOMO) and the lowest unoccupied molecular orbital (LUMO) of Schiff base were -9.16 and -1.256 eV, respectively. It was observed that HOMO of Schiff base focused on benzene. However, the LUMO of Schiff base was dispersed, not only focused on benzene (Fig. 6), but also other group such as carbonyl.

In addition, the bond length and bond angle of Schiff base optimized was also obtained and listed in Table- 1 . The bond length between atoms at imine group is shorter than that at other group. Similarly, the difference between bond angle of atoms at imine group is smaller than that of other group.

\section{Conclusion}

Schiff base derived from $p$-chlorobenzaldehyde and $p$ aminobenzoic acid was synthesized by jet milling and the struc ture was confirmed via ${ }^{1} \mathrm{H}$ NMR and FTIR. Optimized structure of Schiff base was carried out by the program VAMP, the results showed the HOMO and LUMO of Schiff base and the position of Schiff base HOMO and LUMO.

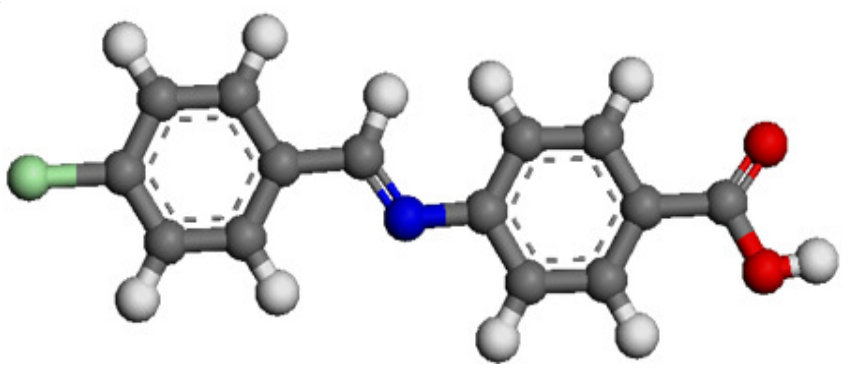

Fig. 5 Optimized geometrical structure of Schiff base 


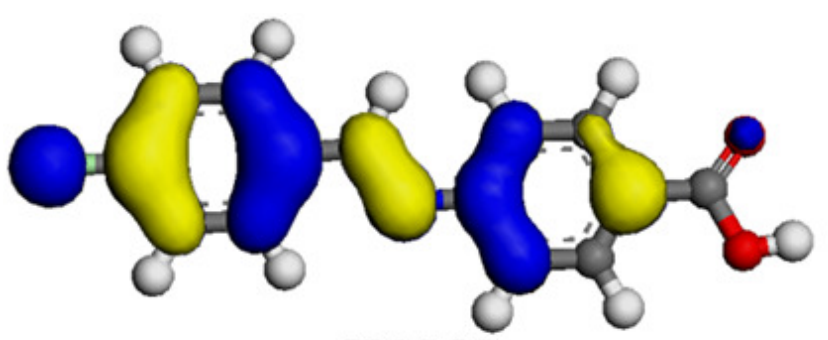

HOMO

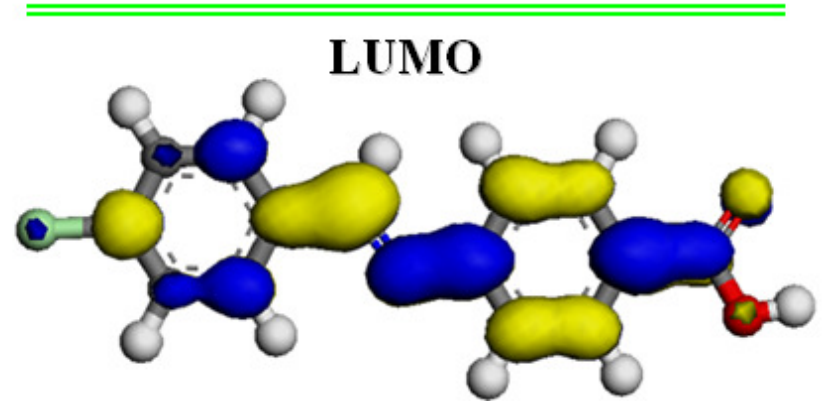

Fig. 6. Frontier molecular orbital of Schiff base

\section{TABLE-1}

BOND LENGTH AND BOND ANGLE OF SCHIFF BASE

\begin{tabular}{cccc}
\hline & Bond length $(\mathrm{nm})$ & & Bond angle $\left(^{\circ}\right)$ \\
\hline $\mathrm{C} 4-\mathrm{H} 5$ & 1.109 & $\angle \mathrm{C} 2 \mathrm{C} 5 \mathrm{H} 4$ & 117.289 \\
$\mathrm{C} 4-\mathrm{N} 6$ & 1.297 & $\angle \mathrm{H} 4 \mathrm{C} 5 \mathrm{~N} 6$ & 121.486 \\
$\mathrm{C} 2-\mathrm{C} 5$ & 1.465 & $\angle \mathrm{C} 5 \mathrm{~N} 6 \mathrm{C} 7$ & 121.858 \\
$\mathrm{~N} 6-\mathrm{C} 7$ & 1.432 & $\angle \mathrm{N} 6 \mathrm{C} 7 \mathrm{C} 8$ & 123.035 \\
& & $\angle \mathrm{N} 6 \mathrm{C} 7 \mathrm{C} 9$ & 116.938 \\
& & $\angle \mathrm{C} 1 \mathrm{C} 2 \mathrm{C} 5$ & 118.052 \\
& & $\angle \mathrm{C} 3 \mathrm{C} 2 \mathrm{C} 5$ & 122.341 \\
\hline
\end{tabular}

\section{ACKNOWLEDGEMENTS}

This work was supported by National Natural Science Foundation of China (Project No. 51403027), China Postdoctoral Science Foundation (Project No. 2013M531937), Scientific and Technological Research Program of Chongqing Municipal Education Commission (Project No. KJ131202) and Chongqing University of Arts and Sciences (Project No. R2012CH10).

\section{REFERENCES}

1. Z. Junfeng and L. Chunshen, J. Fuzhou Univ., 27, 110 (1997).

2. P.J. Mcguiness, E. Devlin, I.R. Harris, E. Rozendaal and J. Ormerod, J. Mater. Sci., 24, 2541 (1989).

3. X. Lu, L. Zhu, C. Liu, L. Zhang, M. Wu and X. Qu, Rare Met., 31, 1 (2012).

4. S.H. Yoon, S.D. Kim, Y. Rang, Y.J. Kim and S.H. Cho, Korean J. Chem. Eng., 12, 436 (1995).

5. I.Y. Saleem and H.D.C. Smyth, AAPS Pharm. Sci. Tech., 11, 1642 (2010).

6. S.J. Li, Asian J. Chem., 23, 2619 (2011).

7. Y.-H. Cai, Asian J. Chem., 24, 4468 (2012).

8. Y.-H. Cai, R.-F. Peng, S.-J. Chu and J.-B. Yin, Asian J. Chem., 22, 5835 (2010). 